Monatsschr Kinderheilkd 2016 $164: 843$

DOI 10.1007/s00112-016-0172-1

Online publiziert: 16 . September 2016

๑) Springer-Verlag Berlin Heidelberg 2016

CrossMark

\section{B. Ure ${ }^{1,2} \cdot$ D. von Schweinitz ${ }^{3}$}

'Medizinische Hochschule Hannover, Hannover, Deutschland

${ }^{2}$ Kinderkrankenhaus Auf der Bult, Hannover, Deutschland

${ }^{3}$ Klinik und Poliklinik für Kinderchirurgie, Dr. von Haunersches Kinderspital, München, Deutschland

\title{
Aktuelle Aspekte und kontroverse Standpunkte der Kinderchirurgie
}

Die Kinderchirurgie weist unter den chirurgischen Disziplinen ein besonders breites Spektrum auf. Wesentlicher Bestandteil dieses Spektrums ist die Chirurgie des Früh-, Neugeborenen und Kleinkindes. Vom Kinderchirurgen fordert die Versorgung dieser Patienten stets ein multidisziplinäres Vorgehen, weshalb die Zusammenarbeit mit Pädiatern unterschiedlichster Bereiche essenziell ist. Diese Zusammenarbeit betrifft neben Neonatologen, pädiatrischen Gastroenterologen, Pulmologen und Nephrologen auch zahlreiche andere Disziplinen der Kinderheilkunde. Aus diesem Grund freuen wir uns sehr, dass uns die Herausgeber der Monatsschrift Kinderheilkunde die Gelegenheit geben, im vorliegenden Heft aktuelle Aspekte zu diesem Themenkomplex darzustellen.

Die Neugeborenenchirurgie selbst ist äußerst umfang- und facettenreich, sodass eine umfassende Diskussion aktueller Aspekte in einem Heft nicht gelingen kann. Deshalb haben wir 5 unserer Meinung nach besonders relevante Themen ausgewählt. Es handelt sich um Bereiche, in denen zahlreiche Aspekte der Diagnostik und der Therapie anhaltend diskutiert werden, häufig zwischen Pädiatern und Kinderchirurgen. Folglich sind in den 5 Beiträgen neben den heutigen Standards auch kontroverse Standpunkte vertreten.

Bespiele für derartige Diskussionspunkte sind chirurgisch technische Aspekte wie der Einsatz minimalinvasiver Operationsverfahren bei der Rekonstruktion der Ösophagus- und Analatresie, der Lungenresektion oder der Korrektur der Hydronephrose. Auch die Dis- kussionen über die Indikation zur chirurgischen Versorgung kongenitaler Lungenmalformationen und die diagnostischen Maßnahmen bei Harntransportstörungen sind nicht abgeschlossen. Aufgrund der Komplexität einzelner Fehlbildungen wie der Analatresie wurden Klassifikationen immer wieder geändert, aktualisiert und auf international einheitliche Standards gebracht. Vor diesem Hintergrund werden in den folgenden Beiträgen wichtige Standpunkte formuliert.

》 Expertise kinderchirurgischer
und multidisziplinärer
Teams entscheidet über das
Behandlungsergebnis

Alle Autoren stimmen darin überein, dass die Expertise kinderchirurgischer und multidisziplinärer Teams entscheidend für das Behandlungsergebnis ist. Komplikationen und funktionelle Langzeitprobleme sind häufig auf mangelnde Erfahrung und niedrige Fallzahlen in einzelnen Kliniken zurückzuführen, weshalb Konsens dahingehend besteht, dass die Früh- und Neugeborenenchirurgie in Zentren durchgeführt werden soll. Eine Zentralisierung wird auch für die interdisziplinäre Nachbehandlung und Langzeitbetreuung als essenziell angesehen, denn die optimale Lebensqualität im Langzeitverlauf erfordert erfahrene Teams und multidisziplinäre Transitionsprogramme.

Die Autoren weisen ebenfalls auf die zunehmende Bedeutung von Patienten- und Elterninitiativen hin. Dies gilt besonders für Erkrankungen der Speiseröhre, des Enddarms oder der Lungen. Der Zusammenarbeit der multidisziplinären Teams in Zentren mit diesen Initiativen wird zunehmende Bedeutung zukommen, besonders im Hinblick auf die Zentralisierung der chirurgischen Versorgung von Neugeboren und Kleinkindern.

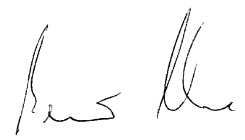

Prof. Dr. B. Ure

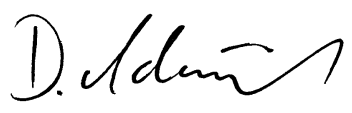

Prof. Dr. D. von Schweinitz

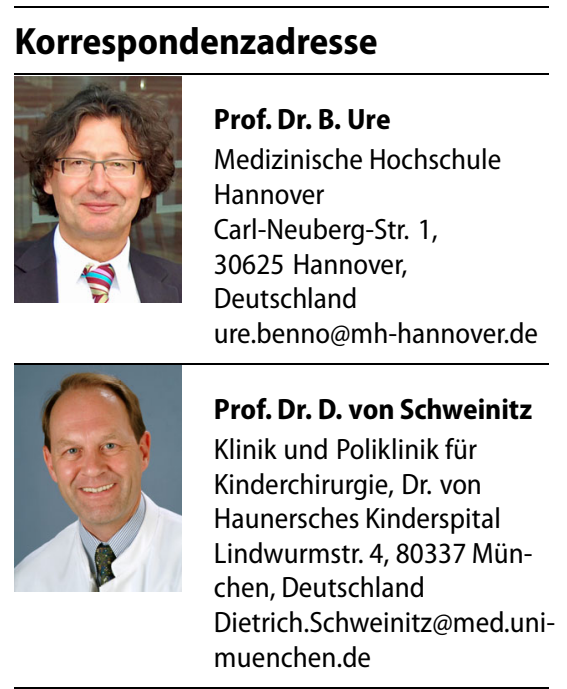

Interessenkonflikt. B. Ure und D. von Schweinitz geben an, dass kein Interessenkonflikt besteht. 